\title{
The Structure of Overionized Plasma in SNR IC 443
}

\section{Bing Jiang ${ }^{1}$, Fabrizio Bocchino ${ }^{2}$, Marco Miceli ${ }^{2}$, Eleonora Troja ${ }^{3}$, Yang Chen ${ }^{1}$ and Hiroya Yamaguchi ${ }^{4} \dagger$}

\author{
${ }^{1}$ Department of Astronomy, Nanjing University, Nanjing 210093, P.R.China \\ email: bjiang@nju.edu.cn \\ ${ }^{2}$ INAF-Osservatorio Astronomico di Palermo, Piazza del Parlamento 1, 90134 Palermo, Italy \\ ${ }^{3}$ NASA, Goddard Space Flight Center, Greenbelt, MD 20771, USA \\ ${ }^{4}$ Harvard-Smithsonian Center for Astrophysics, 60 Garden Street, Cambridge, MA 02138, USA
}

\begin{abstract}
During the last few years, overionized (recombining) plasmas were unexpectedly discovered in a few supernova remnants, but the origin is still unclear. In this contribution, we present a preliminary spectroscopic analysis of the X-ray emission from the north central region of IC443, one of the "recombining" remnants. An overionized NEI plasma model can reproduce well the Ly-alpha lines and the recombination edges in the spectrum. The ionization temperatures for the metals $\mathrm{Mg}$, Si and $\mathrm{S}$ are much higher than the electron temperatures. which is a strong indication of overionization of these elements. The different spectral features of the recombining plasma are characterized on scales of a few arcmin, such as the increasing trend of the pre-cooling temperature and the ionization time from south to north, which may imply a pre-heating direction.
\end{abstract}

Keywords. ISM: individual (IC 443 (G189.1+3.0)), radiation mechanisms: thermal, supernova remnants, X-rays:ISM

\section{Introduction}

In recent decade, the overionized (recombing) plasma was discovered in several supernova remnants (SNRs), such as IC 443 (Kawasaki et al. 2003, hereafter K03; Yamaguchi et al. 2009, hereafter Y09), W49B (Ozawa et al. 2009, Miceli et al. 2010), G359.1-0.5(Ohnishi et al. 2011), W44 (Uchida et al. 2012) and W28(Sawada \& Koyama 2012), challenging the understanding of the evolution of SNRs and its origin is still controversial. IC $443($ G189.1+3.0), in which the overionization was first detected (K03), presents strong recombing emission with Suzaku (Y09) while the XMM-Newton observation suggested only marginal overionized plasma (Troja et al. 2008). With new XMMNewton observation we investigated the difference and the spatial resolved structure of the plasma.

\section{Data Analysis and Results}

Our XMM-Newton observation of IC 443 was performed on 2010 March 07 to March 09 (Obs-ID = 0600110101, PI: E. Troja). The observation data files (ODF) were reprocessed using the XMM-Newton Science Analysis System (SAS) version 11.0.0 task following the standard procedures and were screened for the flares using the XMM-Newton Extended Source Analysis Software (XMM-ESAS) version 4.3.

$\dagger$ Supported by the NSFC grants 11203013 and 11233001, and the grants 20110091120001 and 20120091110048 from the Educational Ministry of China 

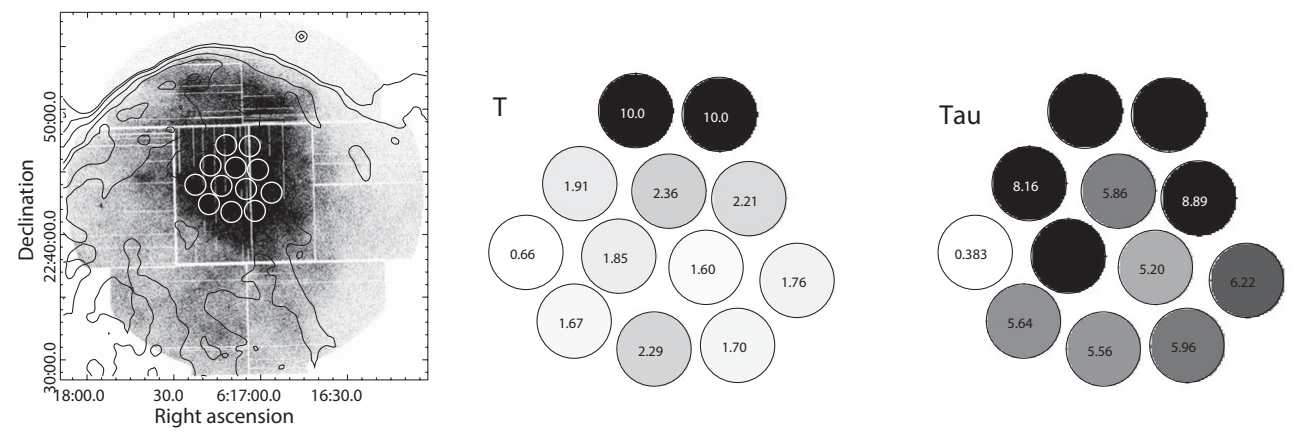

Figure 1. Left: Counts image of IC 443 (northern part) observed by XMM-Newton MOS2 in the $0.3-10 \mathrm{keV}$ band, shown on an intensity color scale, superposed with VLA radio continum at $1.4 \mathrm{GHz}$ (contours) and the 12 regions (circles). Middel: the pre-cooling temperatures obtained from the overionized NEI plasma model for 12 regions in units of $\mathrm{keV}$. Right: the same of the ionization timescales in units of $10^{11} \mathrm{~s} / \mathrm{cm}^{3}$.

We searched for overionized plasma and successfully found it in the north central area of the remnant, around where the overionization was previously detected by K03 and Y09. This area was then divided into 12 arcmin-scale regions for further analysis (Fig. 1).

For all 12 regions, an overionized NEI plasma model can reproduce well Ly-alpha lines and the recombination edges in the X-ray spectra. Using the collisional ionization equilibrium model with one temperature component or two temperature components, the spectra can also be fitted with good $\chi^{2}$ value, however, the Ly- $\alpha$ lines, especially of $\mathrm{Si}$ and $\mathrm{S}$, are failed to be explained. We obtained the ionization temperatures for different metals with that of $\mathrm{Mg}, \mathrm{Si}$ and $\mathrm{S}$ much higher than the electron temperature of the gas, strongly indicating of overionization of these elements. These results are roughly consistent with that in Y09. The electron temperatures obtained from the overionized NEI model, are almost uniform, around $0.5 \mathrm{keV}$, over all regions, while the distributions of the pre-cooling temperatures (ionization temperatures) and the ionization timescales (see Fig. 1) both indicate an increasing trend from south to north, which is probably the pre-heating direction.

\section{References}

Kawasaki, M. T., Ozaki, M., Nagase, F., Masai, K., Ishida, M. \& Petre, R. 2003, ApJ, 572, 897 Miceli, M., Bocchino, F., Decourchelle, A., Ballet, J., \& Reale, F. 2010, A\&A (Letters), 514, L2 Ohnishi, T., Koyama, K., Tsuru, T. G., Masai, K., Yamaguchi, H., \& Ozawa, M. 2011, PASJ, 63,527

Ozawa, M., Koyama, K., Yamaguchi, H., Masai, K., \& Tamagawa, T. 2009, ApJ (Letters), 706, L71

Sawada, M. \& Koyama, K. 2012, PASJ, 64, 81

Troja, E., Bocchino, F., Miceli, M., \& Reale, F. 2008, A\&A A, 485, 777

Uchida, H., Koyama, K., Yamaguchi, H., Sawada, M., Ohnishi, T., Tsuru, T. G., Tanaka, T., Yoshiike, S., \& Fukui, Y. 2012, PASJ, 64, 141

Yamaguchi, H., Ozawa, M., Koyama, K., Masai, K., Hiraga, J. S., Ozaki, M., \& Yonetoku, D. 2009, ApJ (Letters), 705, L6 